\title{
TG23
}

\section{Reservoir Quality Assessment of Tight Gas Reservoirs - Links to Producibility}

\section{R. Klimentidis* (ExxonMobil Research Co.)}

\section{SUMMARY}

Quantification of porosity types and sizes coupled with in-situ reservoir capillary pressure data allows one to estimate the potential hydrocarbon pore volume in a hydrocarbon transition zone. This data coupled with economic gas rate, production and reservoir quality data (such as sandstone compositional and textural trends on a field or basin scale) can provide a tool to evaluate Conventional vs Tight-Gas zones in a prospect.

Conventional gas reservoirs consist predominantly of primary intergranular porosity with large pore-throat sizes and varying amounts of secondary porosity. In contrast, tight-gas reservoirs (i.e. a reservoir which requires artificial stimulation to produce at economic rates) consist predominantly of secondary porosity with pore-throat sizes well below 1 micron in diameter. 
Quantification of porosity types and sizes coupled with in-situ reservoir capillary pressure data allows one to estimate the potential hydrocarbon pore volume in a hydrocarbon transition zone. Integration of this data with economic gas rate production and reservoir quality data (such as sandstone compositional and textural trends on a field or basin scale) can provide a tool to evaluate Conventional vs. Tight-gas zones in a prospect.

Three modes of porosity volumes can be described in sandstones: 1) movable or maximum potential hydrocarbon pore volume usually associated with intergranular porosity; 2) clay-bound water volume associated with detrital and diagenetic clays; and 3) other bound-water typically located in secondary pores within partially-dissolved minerals (i.e. feldspars) and in detrital lithics (sedimentary, volcanic, metamorphic).

The two main porosity types found in sandstone are primary intergranular porosity and secondary microporosity. Primary intergranular porosity occurs between detrital grains. Secondary porosity is located in partially dissolved minerals, microporous detrital grains and matrix, and various diagenetic minerals such as clay minerals (chlorite, kaolinite, illite/smectite, illite etc.). Differentiation and quantification of various porosity types is an essential step in understanding and predicting the producibility of tight-gas reservoirs. MicroQuant, is an SEM/BSE technique which has been developed to quantify secondary microporosity from petrographic thin sections.

Conventional gas reservoirs consist predominantly of primary intergranular porosity with large porethroat sizes and varying amounts of secondary porosity. In contrast, tight-gas reservoirs (i.e. a reservoir which requires artificial stimulation to produce at economic rates) consists predominantly of secondary porosity with pore-throat sizes well below 1 micron in diameter.

Pore-throat size distribution is a key control on properties such as permeability, water saturation, producible pore volume and producibility potential (e.g. hydrocarbon flow rates and cumulative production). The ability to characterize and quantify pore types and sizes based on mineralogical trends in a stratigraphic framework within a basin allows one to build a predictive reservoir quality spatial model. The integration of rock quality data, pore-throat size distribution and economic gas rates can be mapped to differentiate Conventional vs. Tight-gas reservoirs in a basin or field. This protocol can also be used to locate better reservoir quality intervals ("sweet-spots") for optimized field development.

Preliminary results of high-resolution X-Ray Micro-Tomography on tight-gas sandstones supplemented with backscattered electron images, suggest that permeability in very tight sandstones (i.e. permeability below $0.1 \mathrm{mD}$ ) is primarily controlled by the small pore-throats in the clay cements between microporous and non-microporous detrital grains. This suggests that permeability will be dependent on the nature of this pore-fill cement. 